\title{
The Role of Brainstem Sensitization in the Pathophysiology of Deformational Plagiocephaly
}

\section{Rene J Zweedijk*}

Member of the Dutch Osteopathic Association, Director of Pro-Osteo, Responsible for the Osteopathic Pediatric Education Netherlands, The Netherlands

*Corresponding Author: Rene J Zweedijk, Member of the Dutch Osteopathic Association, Director of Pro-Osteo, Responsible for the Osteopathic Pediatric Education Netherlands, The Netherlands.
Received: January 21, 2021

Published: January 30, 2021

(C) All rights are reserved by Rene J Zweedijk.

\begin{abstract}
Deformational Plagiocephaly (DP) is a common clinical presentation. There is no consensus as to the aetiology of DP, there is no risk factor that is uniformly considered as dominant. The consensus about the pathogenetic factors that are important in the onset of DP is more robust. Most cases are presented with no skull deformity at birth and most problems seem to appear at the age of two months. This implies that postnatal factors have an influence on the onset of DP. Prolonged back-laying position in combination with a restriction in motion of the upper neck area are generally considered as important factors in the onset of DP. The restriction in motion can be caused by muscular and neurological reasons. The purpose of this article is to present an aetiology model as to the neurological aspects that may be causing plagiocephaly and how osteopathy may potentially influence the amelioration of plagiocephaly. Sensitization of the upper neck and brainstem may be caused by intrauterine positioning, traumatic birth processes or prolonged pressure on specific areas of the skull. This sensitization will increase sensitivity of touch-, pressure- and pain-fibers, muscle tone, impair muscle function and enhance postural and birth survival reflexes. The postural reflexes, in combination with impaired muscle function and the prolonged supine positioning of the infant, play a crucial role in the progress of some types of Deformational Plagiocephaly (DP). These neurological considerations should be analyzed and treated by osteopaths and allied professions when faced with DP.
\end{abstract}

Keywords: Deformational Plagiocephaly; Brainstem; Aetiology

\section{Introduction}

Plagiocephaly is characterized by an asymmetrical distortion of the skull and it is frequently seen in osteopathic practice [1]. It can be divided into synostotic and non-synostotic variants. The synostotic variant is caused by an absence or a premature closure of one or more cranial sutures and it is often part of a general syndrome [2]. In this case osteopathy is not considered as primary treatment option. The occurrence of non-synostotic plagiocephaly (n-PC) is more frequent than the synostotic variant and osteopathy can be indicated.

Non-synostotic Plagiocephaly (n-PC) is termed Deformational Plagiocephaly (DP) or Postural Cranial Deformity, and is characterized by the absence of ossification changes, but it is linked to functional imbalances in the body [3]. It refers to altered cranial shape in infants older than 6 weeks of age, when the moulding pro- cess of birth is over [1]. DP has a large co-morbidity, has cosmetic consequences and possible consequences for cognitive functioning in later life $[3,4]$.

There are several possibilities for the classification of DP. The most common classifications are the Captier and the Argenta classifications [5,6]. Captier divides DP in Type Fronto-Occipital Plagiocephaly (FOP), Occipital Plagiocephaly (OP) and Posterior Brachycephaly (PB). The Argenta classification scale contains more subtypes of DP [5]. Argenta divides plagiocephaly into 5 subtypes; I,II,III, IV,V. and Posterior Brachycephaly into three subtypes: I,II and III [5]. DP is frequently clinically treated by osteopaths [7]. The osteopathic treatment consist the treatment of strain patterns of the cranial base, treatment of intra- and extracranial membranes, cranial sutures and alignment of the spine [7]. Although the results of the osteopathic treatment seem to be good, there is a lack of 
consensus as to what is causing plagiocephaly and how osteopathy may possibly influence this condition $[2,3,6,7]$.

In a systematic review conducted by De Bock suggests that the etiology of DP is fragmentary and heterogeneous, there is no risk factor that is uniformly considered to be dominant, although male gender, supine sleeping position, limited neck rotation or preference in head position, first born child and lack of tummy time, are frequently-mentioned as risk factors [3].

The consensus on which pathogenetic factors are important in the onset of DP is more robust.

Different forms of DP seem to have different pathogenetic processes. Generally DP is not present at birth and this implies that postnatal factors have an influence on the onset of DP [8]. Since 1990, many children were placed on their backs during sleeping because of the "Sudden Infant Death Syndrome" prevention campaign and resulted in an increase of incidence of DP [9]. Back laying sleeping position in combination with positioning during feeding, sitting for long stretches in car seats and a lack of tummy time or side lying position, may have contributed on the development of DP. This does not explain why some children do develop DP and others don't. Most studies describe the influence of an impaired mobility of the upper neck in combination with supine positioning as one of the main causes $[1,3,6,8]$. The impaired mobility of the upper neck can be due to muscular and neurological reasons [6,8].

Muscular reasons can be divided into:

- $\quad$ Congenital muscular torticollis (CMT), is a unilateral fibrosis of the Sternocleidomastoid muscle (SCM), mostly with a palpable swelling (pseudotumor) of the SCM. CMT can be caused by a) intrauterine/perinatal compartment syndrome or b) disturbed fetal muscular development [10].

- SternoCleidoMastoid (SCM )imbalance. Han calls this a nonlesional torticollis due to the fact that there are no changes in the tissue of the SCM [11]. There is a limit in active rotation and head tilting (side bending), the passive mobility is better than in CMT, there is no swelling of the SCM but the muscle feels hypertonic. In muscle power testing, the muscle is weaker than the opposite side. In this group we see the most severe asymmetry in posture, mainly when the child is brought to an upright position [12]. There is no good explanation of what is causing the SCM imbalance. Figure 1 shows the tilt test which is a reliable test in diagnosing SCM imbalance [12].
The neurological reason for cervical spinal motion restriction due to torticollis caused by neurogenic hypertonia, is not well described and possibly less well-understood. In a small group there is a spasmic form of torticollis due to damage to the central nervous system but mostly there is no pathology present. Of course, other reasons like Sandifer Syndrome, oropharyngeal abscess or tumors must be considered [13]. Osteopathy tries to restore the self-healing force of the human body, partly by increasing mobility and lowering nociceptive stimulation [14]. The osteopathic treatment of DP is not only to improve the cranial deformation(which is, in DP, secondary) but mainly to influence factors that are important in the onset of DP.

The aim of this article is to present a new understanding of the pathogenesis of DP. The role of sensitization of the brainstem is reviewed. As well as the sensitization of the brainstem as a possible cause of SternoCleidoMastoid imbalance and neurogenic torticollis and their influence on the onset of DP is discussed. The article also discussed the implications for Osteopathy.
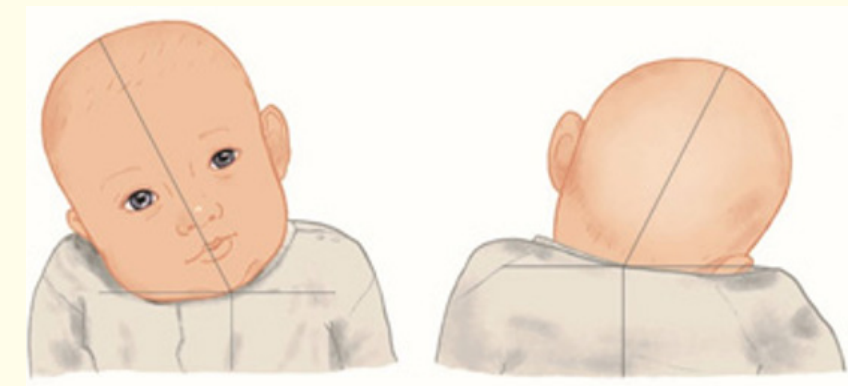

Figure 1: This figure shows the typical position of an infant in the orthopedic SCM function test. From a sitting(supported) position the infant is slightly brought backwards. In case of a SCM dysfunction the head is in a ipsilateral side bending(tilt), light contralateral rotation $[11,12]$.

\section{Sensitization}

Neurogenic torticollis and SternoCleidoMastoid imbalance might be related to sensitization of the nervous system. Sensitization is a reaction to damage or disease of organs or deep somatic tissues. Sensitization can be divided into peripheral and central sensitization and may be an important factor in the onset of DP [15]. 
Peripheral sensitization describes changes in the peripheral tissue and mainly in the nociceptive afferent neurons. This leads to an increase of sensations, called sensitization. Peripheral sensitization can occur as a result of inflammation due to the production of pro-inflammatory cytokines and other chemical mediators such as bradykinin and histamine. This leads to depolarization or sensitization of nerve receptors, Also there can occur an upregulation of receptors due to changes in the dorsal root ganglion [15]. Low threshold stimuli can lead to excitation or normal, non-painful stimuli, can cause pain [16,17]. Peripheral sensitization leads to an increase of temperature sense, while central sensitization does not do this [15].

Central sensitization describes changes in the second order neurons, e.i., interneurons and projecting tract neurons [18].

\section{Sensitization at the level of the brain stem}

In the regulation of the neurological processes in the cranial and the upper neck area, the brain stem is an important structure. On examination, the segmentation of the brain stem (caudal and rostral medulla, pons and lower part of the mesencephalon) is poor, but functionally, there are "hierarchical levels". Processes and reflexes can be organized at the medullary level, e.i. baro-reflexes, at medullary/caudal pontine levels, e.i. blinking reflex or at rostral mesencephalic/pretectal, e.i. the light reflex of the pupil. These levels can be seen as hierarchical levels in this part of the CNS. These levels can be modulated by higher centres such as the hypothalamus and cortex, e.i. as seen in swallowing and the emptying of the stomach.

Neuhuber and Böhni describe the organization of the upper neck and cranial base innervation, the role of the upper cervical nerves and cranial nerves and the important integrative function of the brainstem nuclei $[19,20]$.

Important nuclei in the brain stem are:

- $\quad$ Sensory nuclei, which can be divided into: somatosensory (including nociception) e.i. the trigeminal nucleus; proprioceptive e.i. mesencephalic trigeminal nucleus; and interoceptive/viscerosensory nuclei e.i. solitary tract nucleus $[19,20]$.

- Branchiomotor nuclei, motor nuclei to muscles of the branchial arches (trigeminal, facial, ambiguus Nuclei, and the spinal accessory nucleus (which several authors classify as being Branchiomotor)) for the innervation of masticatory, facial, pharyngo-laryngeal and some neck muscles [19,20].

- Somatomotor nuclei for the innervation of extraocular muscles and tongue
- Parasympathetic nuclei, The Edinger Westphal nucleus of the N. III, Upper Salivatory nucleus of the N.VII, Lower Salivatory nucleus of the N.IX and Dorsal Vagal nucleus of the N. X; parasympathetic neurons for heart and bronchi are located in the external formation of the ambiguus nucleus [19].

Nociceptive information projecting on the upper cervical spinal cord and the brainstem comes from different nerves:

- Ventral and dorsal ramus of the 2 and $3 \mathrm{~d}$ cervical spinal nerves. The ligaments, capsule of the vertebral joints and the upper neck muscles are very densely innervated and are important source of nociceptive stimuli. Lekhel describes that vibration of the neck muscles directly influences postural control [21].

- $\quad$ All three branches of the trigeminal nerve(N.V) are responsible for nociceptive innervation of the intracranial dura mater (DM) [22].

- $\quad$ N. VII, IX, X (dura of the posterior cranial fossa), XI and XII (via ansa cervicalis profunda) [23]. Neuhuber also describes that vagal afferents from the pharynx, the larynx, facial afferents coming from the ear, glossopharyngeal afferents from the tongue also project onto the spinal trigeminal nucleus of the brainstem [19].

Afferent and efferent nerves are related, there is a strong, reflex interaction between several nuclei. Neuhuber describes the interaction between proprioceptive information coming from the upper neck and jaw with the motor function of these areas [19]. Zhang describes the communication between proprioceptive information coming from the jaw and visceral and laryngeal functioning $[24,25]$. Xiong describes that upper neck afferent information also influences the trigeminal nucleus [26]. The trigeminal spinal nucleus is a collection point for information coming from chemothermo- and nociceptive afferent information. The spinal nucleus has a strong connection with both somato- as branchio-motor function, important for normal integrative functioning of the head, jaw, throat and upper neck area. An important hub is the paratrigeminal nucleus (PTN), neuron clusters embedded in the spinal trigeminal tract, which was studied mainly in rats but also in guinea pigs and cats [27]. The existence of the oculo-cardiac reflex, the trigeminal-cardiac reflex and the gag reflex, show that there is a reflex-loop between somato-sensory and parasympathetic centres [28].

Any disease and/or tissue damage which leads to excitation of nociceptive afferents, in osteopathic terms dysfunctions, in the internal or external cranium, in the upper neck, throat and jaw area, 
can cause a peripheral and or central sensitization at the level of the brainstem $[19,29]$. The sensitization is based on the interrelationship of the afferent and efferent fibers and their nuclei [16].

In adults "tension headache", migraine and "Whiplash Associated Dysfunctions" are examples of problems that are associated with peripheral and/or central sensitization in the brainstem and mesencephalon [30].

In newborn babies, birth-trauma or a postnatal irritation of the head and or the upper neck, are putative factors that can lead to this peripheral and central sensitization [31]. There is evidence that irritation of the upper neck due to traction or rotation forces during birth can easily irritate the densely innervated tissues in the cranial base and the upper neck [31]. The ongoing pressure of the bed on the skull, in a baby that is constantly lying on its back, can irritate nociceptive neurons in the cranial base and upper neck [8]. Also the suction force of a vacuum delivery or the resulting damaged area of an associated cephalic hematoma, can cause nociceptive activation and can lead to peripheral and central sensitization [32]. These are a few examples from a long list of putative factors causing sensitization of the brain stem in babies [33].

Pain and/or dysfunction in the upper neck and cranial region can lead to peripheral and/or central sensitization in the brainstem area, causing:

- Impaired muscle function, increased muscle tone [20]

- Increased sensitivity of touch, pressure and pain fibers

- Increased reflex activity

- $\quad$ Altered autonomic function $[20,29]$

These changes will occur in those parts of the brainstem that are mainly affected by the sensitization. For example irritation of the posterior cranial fossa, innervated by superior cervical nerves and $\mathrm{X}$, will tend to influence the nucleii in the lower parts of the brainstem [30].

Sensitization of the lower and rostral medulla, pons and parts of the mesencephalon can cause:

- Increased muscle tone, impaired muscle function; can influence facial muscles (n. VII), eye muscles (nn. III, IV, VI), masticatory muscles (n.V),pharyngeal muscles (n.IX), pharyngeal, laryngeal muscles (n.X), neck muscles, such as descending trapezius, sternocleidomastoid (n.XI) and tongue muscles (n.XII) $[20,34,35]$. There will be an increase in depolarization in EMG $[36]$.
- Increased sensitivity; can influence sensory organs like eyes and ears, giving sono- and photophobia [17,37]. Moreover, the sensitivity of the skin of the skull, the face, the throat, the inner ear, larynx etc. can be also influenced and become hypersensory.

- The reflexes of the brainstem; Reflexes that are conducted over the brainstem will be more enhanced [20].

- $\quad$ Altered autonomic functions; the nuclei that is the origin of preganglionic neurons and that come from the brainstem and the midbrain are parasympathetic. The Parasympathetic System function is to regulate organs and its functions in specific way [38]. In general, we can conclude that sensitization increases parasympathetic function in the cranial area, resulting in an increase of secretion of glands and an altered vascularization of the mucosa [39]. The opposite occurs in the gastrointestinal region. With regards to the upper digestive tract, there seems to be a tonic activity of the dorsal vagal complex that can be inhibited by nociceptive information in the case of sensitization [40].

Sensitization of the brainstem and upper cervical area in newborn and young children.

It is described that intra-uterine positioning, a difficult birth, dominant posture after birth, are all examples of which can stimulate nociceptive afferents in the upper neck and internal and external cranium, leading to a peripheral and central sensitization [31].

Sensitization in newborn and young children can lead to:

- Increased muscle tone and impaired muscle function: In the infant we see an increase of tone and an impaired muscle function of muscles in the upper neck and cranial area. Neurogenic torticollis and SternoCleidoMastoid imbalance are the result of this [11]. Other muscles innervated by the brainstem might also be affected, causing drinking and swallowing problems(muscles of the throat and tongue), jaw asymmetry(muscles of mastication) and strabismus (eyes muscles) [20,34].

- Increased sensibility of the neck and cranium structures. Although it seems very logical, the increase of sensibility is not very well described in literature. There is research that is done in the field of migraine and tinnitus, that supports the theory of increased sensibility [17]. The increase of sensibility could cause problems in posture regulation( the child does not "want to lay on it back")and handling (problem with dressing and undressing, sleeping and feeding) because of the decrease in the threshold of pressure and pain fibers. 
- $\quad$ Altered autonomic function can lead to an increase of mucosal and tear production, a common presentation in young infants [33]. The upper digestive tract seems to be inhibited in the case of sensitization [39]. Problems like mild GERD and infantile colic may be caused or influenced by this phenomenon [33].

- Increased reflexes: The baby and young childen have reflexes that are inhibited by the development of the central nervous system and are not present in the adult. Examples are the Rooting reflex, suckling reflex, Moro-reflex and postural reflexes. Most of these reflexes are conducted over the brainstem [41]. These reflexes are stimulated by afferent input in the upper neck and brain stem area [42]. In the case of sensitization these reflexes will be enhanced.

\section{Sensitization and the development of DP}

Peripheral and central sensitization with their effects in the innervated structures is the equivalent of what in osteopathy is named somatic dysfunction $[14,43]$. A somatic dysfunction in the cranium and or upper neck can influence the onset of DP.

A congenital torticollis with fibrosis of the SternoCleidoMastoid muscle can cause some types of DP due to intracranial tensions as described by Captier [6].

Other types of DP are related to neurogenic hypertonia of the muscles of the upper neck and SCM. ${ }^{6}$ The neurogenic hypertonia leads to a torticollis due to an imbalance in muscle tone and function and resulting in an asymmetric position of the head. Golden calls this Sternocleidomastoid Imbalance which is confirmed by Han who states that the muscle is hypertonic but weak [11,12]. When the baby is placed in an upright position and slightly brought into extension through the spine, the head is held at an asymmetric tilt towards the affected side (see figure 2) [44]. The cause of the neurogenic torticollis and the SternoCleidoMastoid imbalance(SCM are not well described and probably less understood. There is enough evidence to state that central and peripheral sensitization at the level of the brain stem is real and that it also can happen in babies and influence their functioning and behavior. Perinatal factors can lead to this sensitization [31]. Sensitization can be unilateral or bilateral. In palpation the muscles of the upper neck will be hypertonic but when tested, weak in terms of power [11].

Next to impaired muscle tone and function, sensitization will also enhance (primitive) reflexes who are richly present in the baby. Primitive reflexes are brainstem mediated, complex, automatic movements which are present at birth and are inhibited by the maturation of the central nervous system in the first 4-8 months [45]. A part of the reflexes are called postural primitive re- flexes [42]. These reflexes are provoked by exteroceptive and proprioceptive information from the upper neck (muscle spindles and joint receptors) and the vestibulum [41]. The normal disappearance by inhibition for these reflexes is at 5-6 months [42]. Sensitization of the brainstem and upper neck will enhance these reflexes and certainly when this is combined with an impaired mobility of the upper neck. A impaired rotation, side bending or flexion/extension in the upper neck region will provoke the reflex. This will have an dominant influence on body posture regulation of the baby. The ongoing pressure due to the prolonged back-laying posture will deform the skull and provoke the development of DP, mostly a few month after birth as described by van Vlimmeren [8].

An asymmetric sensitization might cause a persisting Asymmetric Tonic Neck reflex (ATNR), resulting in a ipsilateral side bending-contralateral rotation posture and this will cause a Fronto Occipital Plagiocephaly or a Occipital Plagiocephaly. Gieycztor describes that these reflexes also can be an etiological factor in the development of scoliosis [45]. In the case of a symmetric(bilateral) sensitization of the upper neck and brain stem will tend to develop a persisting Tonic Labyrinthine Reflex( TLR) causing an hyperextended posture. This prolonged hyperextended posture can cause a symmetric pressure of the posterior part of the skull and might cause the onset of a DP type brachycephaly. Figure 2 shows DP type Fronto Occipital Plagiocephaly(a) and type Posterior Brachycephaly (b) [6].
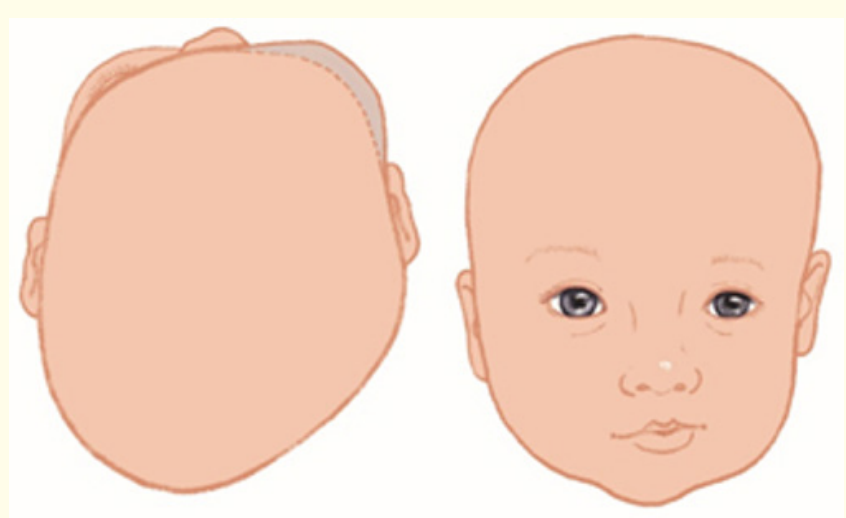

Figure 2: This figure shows a Fronto Occipital Plagiocephaly (a) and a Posterior Brachycephaly(b).

\section{Discussion}

Generally DP is not present at birth and external force (gravity/pressure) is the most important pathogenetic factor $[3,8]$. In combination with the recommended supine sleeping position, a limited range of motion of the upper neck influences these forces. 
The restricted motion of the upper neck may be caused by myogenic or neurogenic torticollis. There are compelling arguments for the proposition that the presented brainstem sensitization model is the scientific base for what is called neurogenic torticollis and SternoCleidoMastoid imbalance. Sensitization is the neurophysiological process of what in osteopathy is called somatic dysfunction. A somatic dysfunction in the upper neck and cranial base area can cause neurogenic torticollis, StrenoCleidoMastoid imbalance and influence the onset of DP.

In some types of DP, the status of the SternoCleidoMastoid (SCM) muscle should be analyzed to exclude a fibrosis of this muscle. This might be a red flag and require referral rather than osteopathic treatment [12]. Sonography can be helpful to diagnose fibrosis of the SCM. When the damage is mild and there is no indication for operation, the torticollis could be treated manually, the same for the, mainly secondary, cranial dysfunctions [46].

When fibrosis of the SCM is not present, sensitization of the brainstem should be considered. The sensitization is characterized by an change in many functions of the upper neck and brainstem area like increased sensibility of touch, pressure and pain fibers, increased muscle tone, decreased muscle function, changed autonomic function and enhanced reflexes [3,47]. In the case of Fronto Occipital Plagiocephaly or a Occipital Plagiocephaly, sensitization is more unilateral, leading to a more asymmetric position of the neck and head and the characteristic asymmetric deformation in form [6]. Often there is a persisting Asymmetric Tonic Neck Reflex (ATNR) due to sensitization of the brainstem nucleii and restric- tion in motion of the upper neck. The sensitization can be caused by dysfunctions in the upper neck and cranial area but will also cause more brainstem facilitation due to an increase of pressure and therefor an increase of nociception [20].

In case of Posterior Brachycephaly, the sensitization is bilateral, giving a dominant Tonic Labyrinthine Reflex (TLR), hyperextension of the neck and deformation in symmetry of the occiput [6]. Here we see very severe symptoms that are not limited to the upper neck and cranium but which influence the whole body (infantile colic, constipation, GERD, etc.) [48].

Figure 3 demonstrates the onset of different type of DP and the role of sensitization of the brainstem.

DP has a large comorbidity, like sleeping problems, drinking problems, gastro intestinal problems, otitis media etc. [3,33,4749]. Sensitization causing an increased sensitivity of the tissues in the cranium and the upper neck in combination with an impaired autonomic function as described before, might be related with this comorbidity [40].

The osteopathic treatment of DP concentrates on the treatment of the deformation of the skull. This deformation is a result of impaired forces after birth [8]. These impaired forces are related with impaired muscle function, enhanced postural reflexes and prolonged back laying position. The impaired muscle function and enhanced reflexes can be the result of brainstem facilitation but will also cause brainstem facilitation and by this vicious circle aggravate the DP.

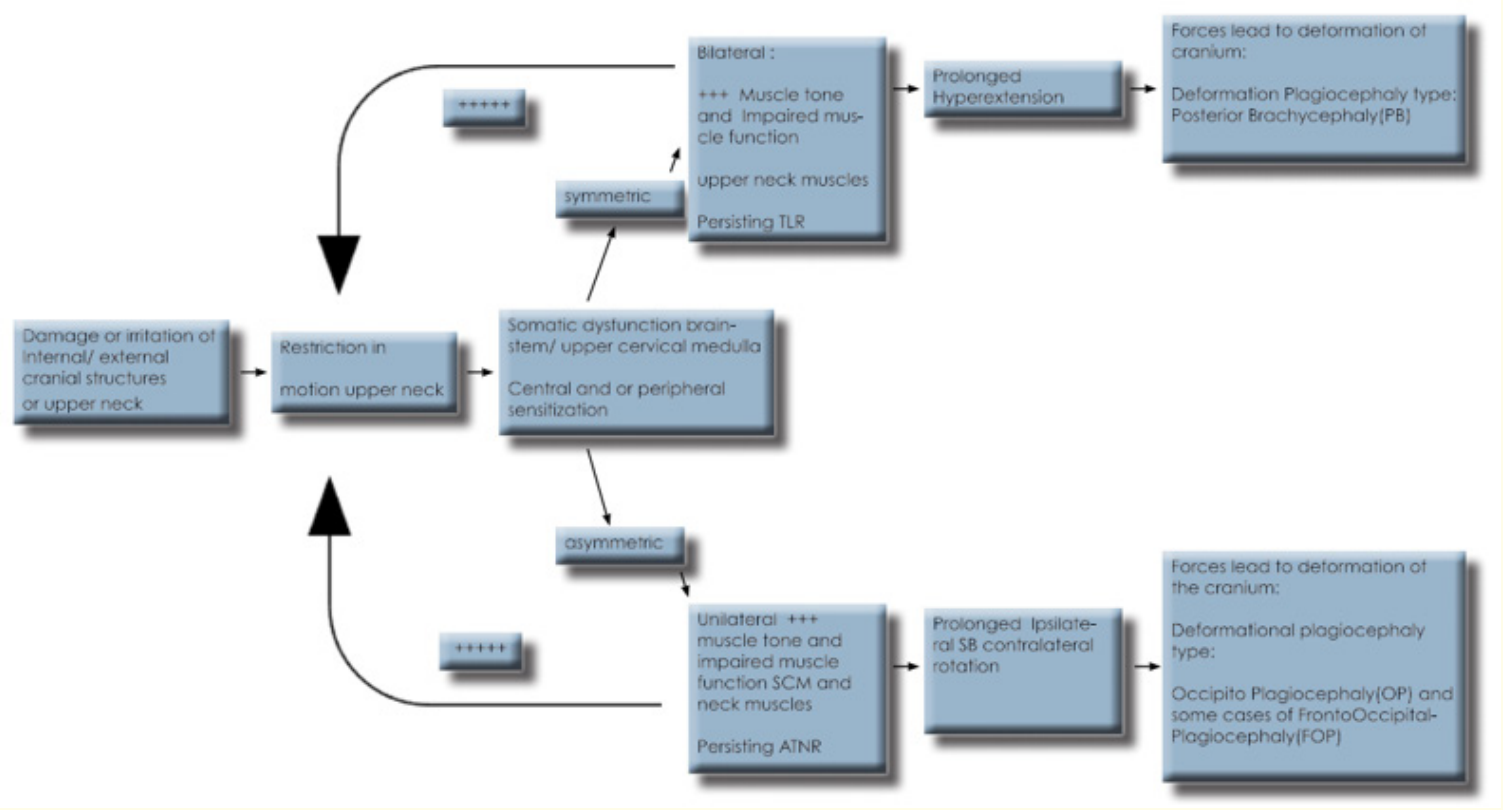

Figure 2: Shows how somatic dysfunction, either symmetric or asymmetric, can be important in the pathophysiology of Deformational Plagiocephaly (DP). Note that one of the main factors are the persisting postural reflexes caused by the somatic dysfunction of the cranium and the upper neck. These postural reflexes cause impaired forces on the cranium and lead to deformation. (TLR: Tonic Labyrinthine Reflex; ATNR: Asymmetric Tonic Neck Reflex; SB: Side Bending). 
Osteopathic treatment restores mobility e.g. of the upper neck vertebra, lowers nociception and thereby lowers sensitization also at the level of the lower brainstem and upper cervical medulla $[14,43]$. The increase in mobility and the related decrease in sensitization, will prevent the child for being in the same posture for many hours of the day. This will spread the forces more over the cranium and prevent the development of DP or even ameliorate the situation. In all types of DP cranial dysfunctions like the different synchondroses, developing sutures and developing cranial bones should be treated [46].

Some authors describe sleeping problems, excessive crying, Gastro Esophageal Reflux Disease, infantile colic, hip displacement as risk factors for DP $[3,47]$. These symptoms might also be caused by an increased sensitivity of the tissues, a changed autonomic (parasympathetic) and somato-motor function caused by sensitization and should not being considered as risk factors but as comorbidity symptoms.

\section{Conclusion}

In this article, the author presents a model that states that peripheral and central sensitization might potentially be the cause of impaired muscle function and enhanced postural reflexes, resulting in an asymmetric tilting or a hyperextension of the upper neck. This can be an important pathogenetic factor in the onset of some types of DP, certainly in combination with prolonged back-laying position.

Next to that the sensitization impairs muscle function and reflex activity, autonomic function and sensibility are also affected. There is evidence that this is responsible for symptoms like GERD, otitis media, handling problems during dressing and washing and infant colic's. Previously these symptoms are described as risk factors but from the view of the sensitization they could be considered as comorbidity symptoms.

Osteopathic treatment of the upper neck and cranial base may lower the sensitization and prevent thereby prevent the onset of DP. Further research to test the hypothesis of the sensitization as a causal factor in the development of some forms of DP is needed. The neurophysiological model as presented in this article needs to be supported by clinical studies. A combination of sonography and superficial EMG is a good option to test the hypothesis in different forms of DP. Another recommendation for further research is to test if the osteopathic treatment of the upper neck area influences sensitization and the onset of DP.

All illustrations are made by Fréderiek Westerweel.

\section{Author Contributions}

Rene J Zweedijk provided substantial contributions to conception and design, acquisition of data, or analysis and interpretation of data. Rene Zweedijk gives final approval of the version of the article to be published; The author agrees to be accountable for all the aspects of the work in ensuring that questions related to the accuracy or integrity of any part of the work are appropriately investigated and resolved.

\section{Bibliography}

1. Lennartsson F and Nordin P. "Nonsynostotic plagiocephaly: a child health care intervention in Skaraborg, Sweden". BMC Pediatrics 19.1 (2019): 48.

2. Ghizoni E., et al. "Diagnosis of infant synostotic and nonsynostotic cranial deformities: a review for pediatricians". Revista Paulista de Pediatria 34.4 (2016): 495-502.

3. De Bock F., et al. "Deformational plagiocephaly in normal infants: a systematic review of causes and hypotheses". Archives of Disease in Childhood 102.6 (2017): 535-542.

4. Collett BR., et al. "Cognitive Outcomes and Positional Plagiocephaly". Pediatrics 143.2 (2019).

5. Argenta L., et al. "Clinical classification of positional plagiocephaly". Journal of Craniofacial Surgery 15.3 (2004): 368-372.

6. Captier G., et al. "Classification and pathogenic models of unintentional postural cranial deformities in infants: plagiocephalies and brachycephalies". Journal of Craniofacial Surgery 22.1 (2011): 33-41.

7. Lessard S., et al. "Exploring the impact of osteopathic treatment on cranial asymmetries associated with nonsynostotic plagiocephaly in infants". Complementary Therapies in Clinical Practice 17.4 (2011): 193-198.

8. van Vlimmeren LA., et al. "Risk factors for deformational plagiocephaly at birth and at 7 weeks of age: a prospective cohort study". Pediatrics 119.2 (2007): e408-418.

9. Cavalier A., et al. "Prevention of deformational plagiocephaly in neonates". Early Human Development 87.8 (2011): 537-543.

10. Xiong Z., et al. "Unique finding in congenital muscular torticollis: Clinic screening on the neck of one day old neonate and ultrasonographic imaging from birth through 3 years of followup". Medicine (Baltimore) 98.11 (2019): e14794. 
11. Han MH., et al. "Comparison of Clinical Findings of Congenital Muscular Torticollis Between Patients With and Without Sternocleidomastoid Lesions as Determined by Ultrasonography". Journal of Pediatric Orthopaedics (2017).

12. Golden KA., et al. "Sternocleidomastoid imbalance versus congenital muscular torticollis: their relationship to positional plagiocephaly". Cleft Palate-Craniofacial Journal 36.3 (1999): 256-261.

13. Herman MJ. "Torticollis in infants and children: common and unusual causes". Instructional Course Lectures 55 (2006): 647653.

14. Hruby RJ., et al. "The five osteopathic models: rationale, application, integration: from an evidence-based to a personcentered osteopathy" (2017).

15. Bolay $\mathrm{H}$ and Moskowitz MA. "Mechanisms of pain modulation in chronic syndromes". Neurology 59 (2002): S2-7.

16. Dux M., et al. "TRP Channels in the Focus of Trigeminal Nociceptor Sensitization Contributing to Primary Headaches". International Journal of Molecular Sciences 21.1 (2020).

17. Noseda R., et al. "Non-Trigeminal Nociceptive Innervation of the Posterior Dura: Implications to Occipital Headache". Journal of Neuroscience 39.10 (2019): 1867-1880.

18. Roldan-Jimenez C., et al. "Central Sensitization in Chronic Musculoskeletal Pain Disorders in Different Populations: A CrossSectional Study". Pain Medicine (2020).

19. Neuhuber W., et al. "Manuelle Medizin 1: Fehlfunktion und Schmerz am Bewegungsorgan verstehen und behandeln". (2015): 357-362.

20. Böhni UW., et al. "Manuelle Medizin 1 : Fehlfunktion und Schmerz am Bewegungsorgan verstehen und behandeln". (2015): 365-372.

21. Lekhel H., et al. "Postural responses to vibration of neck muscles in patients with idiopathic torticollis". Brain 120 (1997): 583-591.

22. Lee SH., et al. "Macroscopic Innervation of the Dura Mater Covering the Middle Cranial Fossa in Humans Correlated to Neurovascular Headache". Frontiers in Neuroanatomy 11 (2017): 127.

23. Lv X., et al. "Innervation of the cerebral dura mater". Neuroradiology Journal 27.3 (2014): 293-298.
24. Zhang J., et al. "Jaw muscle spindle afferents coordinate multiple orofacial motoneurons via common premotor neurons in rats: an electrophysiological and anatomical study". Brain Research 1489 (2012): 37-47.

25. Zhang J., et al. "Monosynaptic circuitry of trigeminal proprioceptive afferents coordinating jaw movement with visceral and laryngeal activities in rats". Neuroscience 135.2 (2005): 497-505.

26. Xiong G and Matsushita M. "Upper cervical afferents to the motor trigeminal nucleus and the subnucleus oralis of the spinal trigeminal nucleus in the rat: an anterograde and retrograde tracing study". Neuroscience Letter 286.2 (2000): 127-130.

27. Saxon DW and Hopkins DA. "Efferent and collateral organization of paratrigeminal nucleus projections: an anterograde and retrograde fluorescent tracer study in the rat". Journal of Comparative Neurology 402.1 (1998): 93-110.

28. Dunville LM and Kramer J. "Oculocardiac Reflex". In: StatPearls. Treasure Island (FL) (2020).

29. King HH., et al. "The Science and Clinical Application of Manual Therapy" (2014).

30. Kontzialis M and Kocak M. "Imaging evaluation of trigeminal neuralgia”. Journal of Istanbul University Faculty of Dentistry 51 (2017): S62-S68.

31. Tekes A., et al. "Birth-related injury to the head and cervical spine in neonates". Magnetic Resonance Imaging Clinics of North America 19.4 (2011): 777-790.

32. Sergueef N., et al. "Palpatory diagnosis of plagiocephaly". Complementary Therapies in Clinical Practice 12.2 (2006): 101110.

33. Ekeus C., et al. "Neonatal complications among 596 infants delivered by vacuum extraction (in relation to characteristics of the extraction)". Journal of Maternal-Fetal and Neonatal Medicine 31.18 (2018): 2402-2408.

34. Andrews MAW. "Stretch Receptor and Somatic Dysfunction: A Narrative Review". Journal of the American Osteopathic Association 119.8 (2019): 511-519.

35. Burstein R., et al. "Neurobiology of Photophobia". Journal of Neuroophthalmology 39.1 (2019): 94-102.

36. Terry EL., et al. "Standardizing procedures to study sensitization of human spinal nociceptive processes: comparing parameters for temporal summation of the nociceptive flexion reflex (TS-NFR)". International Journal of Psychophysiology 81.3 (2011): 263-274. 
37. Noseda R., et al. "Current understanding of photophobia, visual networks and headaches". Cephalalgia 39.13 (2019): 16231634.

38. Travagli RA and Anselmi L. "Vagal neurocircuitry and its influence on gastric motility". Nature Reviews Gastroenterology and Hepatology 13.7 (2016): 389-401.

39. Moller M and May A. "The unique role of the trigeminal autonomic reflex and its modulation in primary headache disorders". Current Opinion in Neurology 32.3 (2019): 438-442.

40. Babic T., et al. "Differential organization of excitatory and inhibitory synapses within the rat dorsal vagal complex". American Journal of Physiology-Gastrointestinal and Liver Physiology 300.1 (2011): G21-32.

41. Shevell M. "The tripartite origins of the tonic neck reflex: Gesell, Gerstmann, and Magnus". Neurology 72.9 (2009): 850853.

42. Gieysztor EZ., et al. "Persistence of primitive reflexes and associated motor problems in healthy preschool children". Archives of Medical Science 14.1 (2018): 167-173.

43. Seffinger MA. American Osteopathic A. "Foundations of osteopathic medicine: philosophy, science, clinical applications, and research" (2018).

44. Sargent B., et al. "Congenital Muscular Torticollis: Bridging the Gap Between Research and Clinical Practice”. Pediatrics 144.2 (2019).

45. Gieysztor EZ., et al. "Trunk rotation due to persistence of primitive reflexes in early school-age children". Advances in Clinical and Experimental Medicine 27.3 (2018): 363-366.

46. Magoun HI., et al. "Osteopathy in the cranial field". [Fort Worth, Tex.]: Sutherland Cranial Teaching Foundation (1997).

47. Oh AK., et al. "Predictors of Severity in Deformational Plagiocephaly". Journal of Craniofacial Surgery 20 (2009): 685-689.

48. Branch LG., et al. "Argenta clinical classification of deformational plagiocephaly". Journal of Craniofacial Surgery 263 (2015): 606-610.

49. Purzycki A., et al. "Incidence of otitis media in children with deformational plagiocephaly". Journal of Craniofacial Surgery 20.5 (2009): 1407-1411.

\section{Assets from publication with us}

- Prompt Acknowledgement after receiving the article

- Thorough Double blinded peer review

- Rapid Publication

- Issue of Publication Certificate

- High visibility of your Published work

Website: www.actascientific.com/

Submit Article: www.actascientific.com/submission.php

Email us: editor@actascientific.com

Contact us: +919182824667 